Gynecol Obstet Invest 1994;38:283-284

\title{
Subject Index Vol. 38, 1994
}

ABO 82 Abortion 241 Adnexalmass $210 \alpha$-Adrenergic receptors 18 Amnion 100, 169 Amniotic fluid 24, 157,169 Anemia 107

Antiestrogenic effects 245 Anti-HIV nucleoside compound d4T, ex vivo transfer 1 Antioxidants 223 Apolipoproteins 10 Arias-Stella's reaction 104 Arm circumference 107 Atrial natriuretic peptide 73

Birth weight 36

Blood group 82

Body mass index 113, 145

Bone metabolism 60

Brain natriuretic peptide 73

Buserelin 249

CA-125 249

Calcitonin 60

Candida albicans 130

Cell adhesion molecules 183

Cerebral arteries 41

Cervical factor 57

Cervix 241

Chemotherapy 14

Child survival 107

Clonidine 18

Collagen 60

Color Doppler sonography 210

- $\quad$ - ultrasonography 96

Computed tomography 28

Continuous-wave Doppler 90

Contraceptives, low-dose, oral 261

Curettage 104

Cyanoacrylate 54

Cytokine 236

Danazol 245 Decidual reaction 104 Diabetes 87 Discordant twin 36 Doppler flow 14

- $\quad$ - velocity waveforms 41

Drug resistance 130

E-cadherin 183 Ectopic pregnancy 104

Emergency section 227 Endometrial biopsy 272

carcinoma 272, 276

changes 104 
polyps 266 Endometriosis 70,183,249 Endometritis 198 Endometrium 183 Endotoxin 169 EPHgestosis 173 Epidermal growth factor 100 Erythrocyte hemoglobin 78 Esophageal varices 45 17ß-Estradiol 253 Estrogen receptors 127,186

replacement therapy 113

Fatty liver 28 Fetal death 87

distress 177

growth 10

- retardation 45, 157

heart rate 177

lung maturity 24 Fibrinolysis 157 Finger blood flow 206 Flow cytometry 227 Folicacid 78

Gestational diabetes mellitus 145

Glucose 41

Growth hormone 145

- binding protein 145

retardation 41

Habitual abortion 223

Height 107

Heparin 5

Hormone replacement therapy 60

Hormones 122

Hot flush 206

Human chorionic gonadotropin 57

decidua associated protein 200217

papillomavirus 134,276

placenta 1

umbilical artery 18 Hyaline membrane disease 87 Hysteroscopy 217,266

Ichthyosis 191 Incontinence 51 Insulin-like growth factor-1 261 Interleukin-1ß 169

Intrauterine growth retardation 177

- $\quad$ insemination 57

Labor induction 163 Lecithin release 100 Leukocytes 191,227 Ligand-binding assay 245 Lipid fractions 223

peroxide 173 Lipoperoxides 223 Liver cirrhosis 45

dysfunction 28 Low-dose aspirin 5

dipyridamole 5 Luteinized unruptured follicle 57 Lymphocyte subtypes 227

typing 227

Malignancy, prediction 140 Malignant gestational trophoblastic

disease 14 Malnutrition 107

Massive interviUous fibrin deposition 5 Maternal health 107 Menopause $206 \alpha$-Methyldopa 18

Micropapillae 134 Monoclonal antibodies 227 Mozambique 82,151,198

Neonatal morbidity 36 Normal human endometrium 186 Notching 90 Nulliparous women 261

Obstructed labor 31

Oligomenorrhea 122

Omentum 213

Oncogenes 70

Ovarian carcinoma $65,140,213,276$ 
- neoplasm 140

Ovulatory disturbance 117

Pacemaker 163 Parturition 241 P-cadherin 183 Pelvic girdle relaxation 21

- pain 21

Pipelle sampling device 272 Plasminogen activator inhibitors 157 Polycystic ovaries 117

Polymerase chain reaction 134,276 Portal hypertension 45

Postmenopause 60, 113

Preeclampsia 28, 73, 157

Pregnancy 10,45,78, 145, 157, 191,241

-, immunity 236

Pregnancy-induced hypertension 90, 236

Premenstrual symptoms 122

Procollagen type I carboxy-terminal

propeptide 60 Progesterone receptors 127,186 Puerperium 51,198 -, septicemia 198

Recurrent fetal growth retardation 5 Relaxin 21

Respiratory distress syndrome 87 Rhesus antigen distribution 82

Sclerotherapy 45 Serum paraoxonase 10 Shunt operation 45 Sonohysterography 266

Spontaneous abortion 104 - delivery 227

Sterilization 54 Steroid receptors 245

- $\quad$ sulfatase activity 191

Steroids 87

Surfactant protein SP-B 24 Sweden 82

Sympathetic nervous system 206 Symphysiotomy 31

Tamoxifen 127 T cells 236 Tissue adhesive 54 Torulopsis glabrata 130 Transvaginal ultrasound

117,210 Triglycerides 10 Tubal anastomosis 54

obstruction 54 Tumor necrosis factor- $\beta 169$

suppressor genes 70

Ultrasound 213

- $\quad$ contrast medium 210

Umbilical cord 96

Urinary retention 51

- $\quad$ trypsin inhibitor 169

Urine 169

Uterine activity 163

distention 163

fluid 217

rupture 151

-, audit 151 Uterus 241

Vagina 241 Vaginal ring 253 Vaginismus 194 Vasodilation 206 Vitamin B12 78

- E 173

Vulvar vestibulitis syndrome 194 Vulvovaginitis 130

Weight 107

Yohimbine 18

284

Subject Index 